\title{
Article \\ Adults' Acceptance of COVID-19 Vaccine for Children in Selected Lower- and Middle-Income Countries
}

\author{
Suzanna Awang Bono ${ }^{1}(\mathbb{D})$, Ching Sin Siau 2,*(D), Won Sun Chen ${ }^{3}\left(\mathbb{D}\right.$, Wah Yun Low ${ }^{4,5}$, \\ Edlaine Faria de Moura Villela ${ }^{6,7}$ (D), Supa Pengpid ${ }^{8}$, M Tasdik Hasan ${ }^{9,10}$, Philippe Sessou ${ }^{11(D),}$ \\ John D. Ditekemena ${ }^{12}$, Bob Omoda Amodan 13 (D), Mina C. Hosseinipour 14,15, Housseini Dolo ${ }^{16}$, \\ Joseph Nelson Siewe Fodjo ${ }^{17,18}$ (D) and Robert Colebunders ${ }^{17}$ (D)
}

1 School of Social Science, Universiti Sains Malaysia, Gelugor 11800, Malaysia; suzanna.bono@usm.my

2 Centre for Community Health Studies (ReaCH), Faculty of Health Sciences, Universiti Kebangsaan Malaysia, Kuala Lumpur 50300, Malaysia

3 Department of Health Science and Biostatistics, Faculty of Health, Arts and Design, Swinburne University of Technology, Hawthorn, VIC 3122, Australia; wchen@swin.edu.au

4 Faculty of Medicine, Universiti Malaya, Kuala Lumpur 50603, Malaysia; lowwy@um.edu.my

5 Asia Europe Institute, Universiti Malaya, Kuala Lumpur 50603, Malaysia

6 Disease Control Coordination, São Paulo State Health Department, Sao Paulo 01246-000, Brazil; edlaine@alumni.usp.br

7 Institute of Tropical Pathology and Public Health, Federal University of Goiás, Goiania 74690-900, Brazil

8 ASEAN Institute for Health Development, Mahidol University, Nakhon Pathom 73170, Thailand; supa.pen@mahidol.ac.th

9 Jeeon Bangladesh Ltd., Dhaka 1207, Bangladesh; tasdikhdip@yahoo.com

10 Department of Primary Care \& Mental Health, University of Liverpool, Liverpool L69 3BX, UK

check for

updates

Citation: Bono, S.A.; Siau, C.S.; Chen, W.S.; Low, W.Y.; Faria de Moura

Villela, E.; Pengpid, S.; Hasan, M.T.;

Sessou, P.; Ditekemena, J.D.; Amodan, B.O.; et al. Adults' Acceptance of COVID-19 Vaccine for Children in Selected Lower- and Middle-Income Countries. Vaccines 2022, 10, 11 . https://doi.org/10.3390/ vaccines10010011

Academic Editors: Vincenzo Baldo and Christian Napoli

Received: 5 November 2021

Accepted: 20 December 2021

Published: 22 December 2021

Publisher's Note: MDPI stays neutral with regard to jurisdictional claims in published maps and institutional affiliations.

Copyright: (c) 2021 by the authors. Licensee MDPI, Basel, Switzerland. This article is an open access article distributed under the terms and conditions of the Creative Commons Attribution (CC BY) license (https:// creativecommons.org/licenses/by/ $4.0 /)$.
11 Research Unit on Communicable Diseases, Polytechnic School of Abomey-Calavi, University of Abomey-Calavi, Cotonou 01 BP 526, Benin; sessouphilippe@yahoo.fr

12 Kinshasa School of Public Health, University of Kinshasa, Kinshasa 7948, Democratic Republic of the Congo; jditekemena@hotmail.fr

13 Uganda Public Health Fellowship Program, Loudel Towers, Level 4, Kampala 7272, Uganda; bomoda@musph.ac.ug

14 School of Medicine, University of North Carolina at Chapel Hill, Chapel Hill, NC 27599, USA; mina_hosseinipour@med.unc.edu

15 University of North Carolina Malawi, Lilongwe 999119, Malawi

16 International Center of Excellence in Research, Faculty of Medicine and Odonto Stomatology, University of Sciences, Techniques and Technology of Bamako, Bamako BP 1805, Mali; dolohousseini@yahoo.fr

17 Global Health Institute, University of Antwerp, 2000 Antwerp, Belgium; josephnelson.siewefodjo@uantwerpen.be (J.N.S.F.); robert.colebunders@uantwerpen.be (R.C.)

18 Brain Research Africa Initiative (BRAIN), Yaounde P.O. Box 25625, Cameroon

* Correspondence: chingsin.siau@ukm.edu.my

\begin{abstract}
Since emergency approval of COVID-19 vaccines for children aged between 12 and 15 years old was recently obtained in the United States and Europe, we aimed to assess the willingness to vaccinate children with a COVID-19 vaccine in lower- and middle-income countries (LMICs). Therefore, we launched an online cross-sectional survey in several LMICs. Questions relating to socio-demographic information, knowledge of COVID-19, level of fear/worry of being infected with COVID-19, and willingness to vaccinate children with the COVID-19 vaccine at $50 \%, 75 \%$ and $95 \%$ effectiveness levels, were asked. Of the 6571 participants (mean age $=39 \pm 14$ years), $64.0 \%, 72.6 \%$, and $92.9 \%$ were willing to vaccinate children at $50 \%, 75 \%$, and $95 \%$ effectiveness levels, respectively. Respondents who were undergraduates, who were more worried/fearful about COVID-19, had higher knowledge scores regarding COVID-19, and a higher belief that COVID-19 vaccination is important to protect others, were more willing to accept COVID-19 vaccination of children. COVID-19 vaccination of children will limit the spread of the virus, especially in schools; it may decrease the need for school closures which has a negative effect on child development. Findings from this study are useful for health promotion strategies during COVID-19 vaccination implementation among children in LMICs.
\end{abstract}


Keywords: COVID-19 vaccination; children; lower- and middle-income countries; parents; caretaker; Brazil; Malaysia; Thailand; Bangladesh; Africa

\section{Introduction}

The coronavirus disease 2019 (COVID-19) was declared a pandemic by the World Health Organization (WHO) on 11 March 2020 and precipitated a global health emergency [1]. As of 9 December 2021, a total of 265,713,467 confirmed cases and 5,260,888 deaths were reported [2]. Against the unmitigated increase of new cases, the rapid authorization and roll out of COVID-19 vaccines serves as an important prevention measure against COVID-19 infection. COVID-19 vaccination is very pertinent to any government's strategy in recovery and achieving herd immunity. Vaccines play a critical role in preventing death and hospitalization caused by infectious diseases [3,4]. Vaccine trials have reported encouraging results indicating that a COVID-19 vaccine is safe and produces a good immune response [5,6]. The Pfizer BioNTech and Moderna vaccines were authorized for emergency use by the Food and Drug Administration in the United States of America in December 2020 for individuals who were 16 years old and older [3]. In the same month, the first COVID-19 vaccine, Pfizer BioNTech, also received emergency authorization in the European Union [7]. COVID-19 vaccines have been shown to be effective in controlling the spread of the disease [4]. Further awareness of the need for vaccination and additional protective behaviors is required to control the pandemic. Among lower-income countries, the COVAX initiative was implemented to enable equitable and accelerated distribution of the vaccines.

As of 7 December 2021, a total of 3.53 billion individuals were fully vaccinated [8]. As the COVID-19 vaccine is being rolled out globally and an increasing number of the adult population is being vaccinated, more interest has been generated in extending COVID-19 vaccination to children. In May 2021, Canada and the United States of America granted emergency approval of the COVID-19 vaccine for use among children aged between 12 and 15 years $[9,10]$.

The success of a vaccination program depends on rates of uptake among the population. Adverse events, especially side effects may have led some people to express concerns about getting vaccinated, delay getting vaccinated or even strongly oppose vaccination, and may have reduced their confidence in national safety monitoring systems. Further, a challenge in communicating the importance of COVID-19 vaccination is that younger adults are typically less clinically affected by COVID-19 infection and so may see limited value in getting vaccinated [11]. In addition, misinformation about future COVID-19 vaccines has circulated on social media platforms [12], further amplified by already high levels of vaccine misinformation in general [13]. Vaccine hesitancy overall has risen so substantially that the WHO now considers it a major threat to global health. A specific challenge for a COVID-19 vaccine is that its expedited development may contribute to the public impression that the vaccine will not have been sufficiently tested for safety and efficacy [13-15].

Several factors have led to hesitancy among individuals and governments to start vaccinating children. Firstly, COVID-19 symptoms are milder and rarely lead to hospitalization and mortality among children, compared to adults [16]. In view of this, especially since only approximately $44.7 \%$ of the global population was fully vaccinated as of 7 December 2021, there is a greater urgency to vaccinate adults [8]. In addition, the safety of COVID-19 vaccines for administration among children is still being established [17].

Compared to a very large number of studies addressing COVID-19 vaccine acceptance among adults [18], there is a limited number of studies examining COVID-19 vaccine acceptance for children especially among low- and middle-income countries (LMICs) [19-21]. Given the feasibility of vaccinating children in LMICs with the COVID-19 vaccine in the near future, our research questions were: (1) What are the COVID-19 vaccine acceptance 
rates for children at 50\%,75\%, and $95 \%$ effectiveness levels, and (2) What are the factors affecting vaccine acceptance for children? In an online survey in three South East Asian countries, Brazil, and several African countries, we investigated willingness to vaccinate children with vaccines of three different levels of effectiveness, $50 \%, 75 \%$, and $95 \%$. The 95\% level was chosen based on the initial results of the COVID-19 messenger RNA (mRNA) vaccines showing an effectiveness of $95 \%$ [22], the $50 \%$ level is the minimum level for a vaccine to be approved [23], and the $75 \%$ level is an intermediate level where vaccination could be considered depending on severity of the disease [24,25]. We hypothesized that the higher the vaccine effectiveness, the higher the vaccine acceptance would be. We also explored the factors that may affect vaccine acceptance for children.

\section{Materials and Methods}

\subsection{Design and Participants}

This was a descriptive cross-sectional study. This study is a part of the International Citizen Project (ICP) COVID-19 (https:/ / www.icpcovid.com/en/form/covid-19-vaccinesurvey, accessed on 4 November 2021) whereby a series of large-scale online surveys are developed to understand country-level adherence to the interventions recommended by the WHO. The English version of the questionnaire was pilot-tested among ICPCovid team members and translated into the main national languages of the participating countries. We targeted adult members of the general public to understand their attitude toward COVID-19 vaccination of children. Participants were recruited based on specific inclusion criteria which were: (1) being at least 18 years old, and (2) providing informed consent to participate in this study. Participants were recruited in LMICs namely, Brazil, Malaysia, Thailand, Bangladesh, and African countries, including the Democratic Republic of Congo, Uganda, and Malawi. Ethical approval for this study was granted by the ethics committees in respective participating countries (see author statements for more details).

\subsection{Materials}

There were three parts to the questionnaire. In the first part of the questionnaire, demographic information (e.g., age, sex, country of residence, educational level, studying or working in healthcare, number of people currently living with according to age, selfperceived socio-economic status, self-perceived area of residence, and working/studying from home) was collected. These variables were chosen as they had been shown in past studies to be associated with vaccine acceptance $[26,27]$. Those who are working or studying from home may be less open to vaccinate children since they are less at risk for contracting the virus in comparison with individuals who are not working or studying from home. In the second part, participants were asked about health status, knowledge of COVID19 , and their level of fear/worry of being infected with COVID-19. With respect to the COVID-19 knowledge items, each "Yes" answer scored 1 point, and each "No" answer scored 0 point. These items were: (1) possibility of being re-infected after recovering from a previous COVID-19 infection, (2) COVID-19 infection could be prevented by a vaccine, and (3) there is currently an effective vaccine against COVID-19. The third and last part of the questionnaire consisted of three questions regarding the willingness of participants to vaccinate children with the COVID-19 vaccine at 50\%, 75\% and 95\% effectiveness levels.

\subsection{Procedure}

The original questionnaire was in English. The research collaborators from the participating countries translated the questionnaire into their own national language. The translated questionnaires were pilot-tested among the team members of the ICPCovid consortium belonging to the respective countries. Self-administered online questionnaires were disseminated through social media platforms, such as WhatsApp, Facebook, Messenger, Twitter, and university webpage portals, between 10 December 2020 and 9 February 2021, by the researchers to their personal and professional networks. Participation was voluntary. Participants were asked to provide informed consent before attempting the 
questionnaire. Participants' information and answers were kept private and confidential by not retrieving any information that could lead the investigators to the participants.

\subsection{Weighting}

Prior to conducting data analysis, all data were weighted. The weighting process was previously described in detail by Bono et al. [28].

\subsection{Statistical Analysis}

Descriptive statistics were presented as means and standard deviations (SD) for continuous variables, and categorical variables were summarized using frequency and percentage. Chi-square tests were used to test the associations between the demographic characteristics and vaccine acceptance at $50 \%, 75 \%$, and $95 \%$ effectiveness levels. Independent samples $\mathrm{t}$-tests were used to test whether there were significant differences in vaccine acceptance at all levels in terms of worry/fearfulness of being infected by COVID-19, total knowledge score, and importance of getting the vaccine to protect others. A series of multiple logistic regression analyses was conducted with vaccine acceptance as the dependent variable, at $50 \%, 75 \%$, and $95 \%$ effectiveness levels. The dependent variable was coded $1=$ yes and $0=$ no/no opinion/not applicable. We verified all assumptions for multiple logistic regression and used the Spearman's Rank correlation coefficient and Pearson's correlation coefficient to investigate the multi-collinearity of the factors. The variable "Importance of taking COVID-19 vaccine to protect self" was excluded from the regression analyses due to its high correlation with "Importance of taking COVID-19 vaccine to protect others". A p-value of less than 0.05 (two-tailed) was deemed statistically significant. All analyses were conducted using SPSS [29].

\section{Results}

A total of 10,183 individuals responded to the questionnaire, of which 6571 (64.5\%; mean age $=39 \pm 14$ ) responded to the question regarding the willingness to vaccinate children. Post-hoc power analysis was conducted using logistic regression in G*Power (version 3.1.2) [30]. Based on the 95\% effectiveness logistic regression model, with $n=6571$, the odds ratio for females in predicting vaccine acceptance for children $=0.55, \mathrm{R} 2$ of other $\mathrm{X}=0.48$, the critical $\mathrm{z}=-1.96$ and power $(1-\beta$ err prob $)=1.00$. Therefore, this study was adequately powered. Of the participants $(n=6571)$, the majority were female $(64.8 \%)$, had postgraduate education $(50.3 \%)$, belonged to the upper-middle income category $(46.0 \%)$, lived in an urban setting (83.6\%), and worked/studied from home (58.4\%) (Table 1). All countries, except the African countries, had more female participants. About one third $(29.9 \%)$ were students/workers in the healthcare sector. In terms of household composition, $30.5 \%$ reported living with children $<12$ years old, whilst $20.5 \%$ reported living with children 12 to 17 years old. Since the beginning of the COVID-19 outbreak, 8.8\% had tested positive for COVID-19 and $36.2 \%$ had tested COVID-19 negative at the time of the survey.

Regarding the participants' willingness to vaccinate children, $64.0 \%(95 \% \mathrm{CI}[62.7$, $65.2]), 72.6 \%(95 \% \mathrm{CI}[71.5,73.7])$, and $92.9 \%(95 \% \mathrm{CI}[92.2,93.5])$ were willing to vaccinate at the $50 \%, 75 \%$, and $95 \%$ effectiveness levels, respectively. However, only $66.4 \%$ of the participants believed that there is currently an effective vaccine against COVID-19. Most of the participants reported that it is extremely important to take the COVID-19 vaccine to protect others $(72.0 \%)$ (Table 2). Chi-square and independent samples $t$-tests revealed significant findings for all variables except residential setting, with vaccine acceptance at $95 \%$ effectiveness level, and healthcare worker/student status, with vaccine acceptance at $75 \%$ and $95 \%$ effectiveness levels (Table 3 ). 
Table 1. Participants' demographics and health status $(n=6571)$.

\begin{tabular}{|c|c|c|c|c|c|c|}
\hline \multirow[t]{2}{*}{ Variable } & Total & $\begin{array}{c}\text { Brazil } \\
n=4867\end{array}$ & $\begin{array}{c}\text { Malaysia } \\
n=1245\end{array}$ & $\begin{array}{c}\text { Thailand } \\
n=122\end{array}$ & $\begin{array}{c}\text { Bangladesh } \\
\quad n=199\end{array}$ & $\begin{array}{c}\text { African } \\
\text { Countries }^{\dagger} \\
n=138\end{array}$ \\
\hline & $n(\%)$ & $n(\%)$ & $n(\%)$ & $n(\%)$ & $n(\%)$ & $n(\%)$ \\
\hline \multicolumn{7}{|l|}{ Demographics } \\
\hline \multicolumn{7}{|l|}{ Gender } \\
\hline Male & $2312(35.2)$ & $1661(34.1)$ & $446.0(35.8)$ & $32.0(26.2)$ & $79.0(39.7)$ & $94.0(68.1)$ \\
\hline Female & $4259(64.8)$ & $3206(65.9)$ & $799.0(64.2)$ & $90.0(73.8)$ & $120.0(60.3)$ & $44.0(31.9)$ \\
\hline \multicolumn{7}{|l|}{ Age, years } \\
\hline Mean \pm SD & $47 \pm 15$ & $49 \pm 14$ & $41 \pm 15$ & $47 \pm 10$ & $29 \pm 6$ & $35 \pm 9$ \\
\hline Median (Min, Max) & $48(18,93)$ & $51(18,93)$ & $38(18,82)$ & $47(24,76)$ & $29(18,60)$ & $35(18,65)$ \\
\hline $18-29$ & $945(14.4)$ & $414(8.5)$ & $357(28.7)$ & $7(5.7)$ & $119(59.8)$ & $48(34.8)$ \\
\hline $30-39$ & $1320(20.1)$ & $895(18.4)$ & $286(23.0)$ & $24(19.7)$ & $72(36.2)$ & $43(31.2)$ \\
\hline $40-49$ & $1254(19.1)$ & $1001(20.6)$ & $172(13.8)$ & $38(31.1)$ & $5(2.5)$ & $38(27.5)$ \\
\hline $50-59$ & $1498(22.8)$ & $1239(25.5)$ & $206(16.5)$ & $45(36.9)$ & $1(0.5)$ & $7(5.1)$ \\
\hline 60 and above & $1554(23.6)$ & $1318(27.1)$ & $224(18.0)$ & $8(6.6)$ & $2(1.0)$ & $2(1.4)$ \\
\hline \multicolumn{7}{|l|}{ Education level } \\
\hline Primary/Secondary & $841(12.8)$ & $580(11.9)$ & $230(18.5)$ & $6(4.9)$ & $17(8.5)$ & $8(5.8)$ \\
\hline Undergraduate Degree & $2425(36.9)$ & $1508(31.0)$ & $652(52.4)$ & $63(51.6)$ & $109(54.8)$ & $93(67.4)$ \\
\hline Postgraduate Degree & $3305(50.3)$ & $2779(57.1)$ & $363(29.2)$ & $53(43.4)$ & $73(36.7)$ & $37(26.8)$ \\
\hline \multicolumn{7}{|l|}{ Socio-economic status } \\
\hline Low/Lower Middle & $3177(48.3)$ & $2206(45.3)$ & $694(55.7)$ & $92(75.4)$ & $82(41.2)$ & 103 (74.6) \\
\hline Upper Middle & $3025(46.0)$ & $2342(48.1)$ & $512(41.1)$ & $26(21.3)$ & $111(55.8)$ & $34(24.6)$ \\
\hline High & $369(5.6)$ & $319(6.6)$ & $39(3.1)$ & $4(3.3)$ & $6(3.0)$ & $1(0.7)$ \\
\hline \multicolumn{7}{|l|}{ Residential setting } \\
\hline Rural & $294(4.5)$ & $111(2.3)$ & $155(12.4)$ & $23(18.9)$ & $3(1.5)$ & $2(1.4)$ \\
\hline Suburban/Slum & $786(12.0)$ & $542(11.1)$ & $182(14.6)$ & $19(15.6)$ & $29(14.6)$ & $14(10.1)$ \\
\hline Urban & $5491(83.6)$ & $4214(86.6)$ & 908 (72.9) & $80(65.6)$ & $167(83.9)$ & $122(88.4)$ \\
\hline Healthcare worker or student (Yes) & $1968(29.9)$ & $1443(29.6)$ & $270(21.7)$ & $49(40.2)$ & $116(58.3)$ & $90(65.2)$ \\
\hline \multicolumn{6}{|l|}{ COVID-19 testing } & $46(33.3)$ \\
\hline Not tested/Don't know test results & $3614(55.0)$ & $2444(50.2)$ & $873(70.1)$ & $103(84.4)$ & $111(55.8)$ & $83(60.1)$ \\
\hline Tested, negative & $2379(36.2)$ & $1893(38.9)$ & $360(28.9)$ & $19(15.6)$ & $56(28.1)$ & $51(37.0)$ \\
\hline Tested, positive & $578(8.8)$ & $530(10.9)$ & $12(1.0)$ & $0(0.0)$ & $32(16.1)$ & $4(2.9)$ \\
\hline \multicolumn{7}{|l|}{ Household } \\
\hline Living with children $<12$ years old & $2004(30.5)$ & $1417(29.1)$ & $395(31.7)$ & $25(20.5)$ & $81(40.7)$ & $86(62.3)$ \\
\hline Living with children 12 to 17 years old & $1350(20.5)$ & $902(18.5)$ & $312(25.1)$ & $21(17.2)$ & $47(23.6)$ & $68(49.3)$ \\
\hline
\end{tabular}

${ }^{\dagger}$ Countries in Africa in this study comprised of DR Congo $(n=117)$, Uganda $(n=8)$, and Malawi $(n=13)$.

Compared to Brazil, all other countries reported lower willingness to vaccinate children, with Thailand having the lowest level of acceptance at 50\% effectiveness (aOR: 0.06, $95 \%$ CI $[0.05,0.08], p<0.001$ ) and 75\% effectiveness (aOR: 0.06, 95\%CI [0.04, 0.08], $p<0.001$ ) (Table 3). However, at $95 \%$ effectiveness level, child vaccine acceptance was only significantly lower in Malaysia (aOR: $0.58,95 \% \mathrm{CI}[0.38,0.88], p=0.011$ ) and the African countries (aOR: $0.23,95 \% \mathrm{CI}[0.16,0.32], p<0.001$ ), compared to Brazil. 
Table 2. Knowledge and attitudes towards COVID-19 vaccination.

\begin{tabular}{|c|c|c|c|c|c|c|}
\hline \multirow[t]{2}{*}{ Variable } & Total & $\begin{array}{c}\text { Brazil } \\
n=4867\end{array}$ & $\begin{array}{c}\text { Malaysia } \\
n=1245\end{array}$ & $\begin{array}{c}\text { Thailand } \\
n=122\end{array}$ & $\begin{array}{c}\text { Bangladesh } \\
n=199\end{array}$ & $\begin{array}{c}\text { African } \\
\text { Countries }^{\dagger} \\
n=138\end{array}$ \\
\hline & $n(\%)$ & $n(\%)$ & $n(\%)$ & $n(\%)$ & $n(\%)$ & $n(\%)$ \\
\hline \multicolumn{7}{|c|}{ Willingness to vaccinate children } \\
\hline At $50 \%$ Effectiveness & $3691(64.0)$ & $3313(77.1)$ & $289(27.2)$ & $15(13.0)$ & $59(34.1)$ & $15(12.4)$ \\
\hline At $75 \%$ Effectiveness & $4432(72.6)$ & $3917(85.2)$ & $383(35.2)$ & $23(20.2)$ & $93(50.0)$ & $16(13.4)$ \\
\hline At $95 \%$ Effectiveness & $6107(92.9)$ & 4667 (95.9) & $1060(85.1)$ & $109(89.3)$ & $185(93.0)$ & $86(62.3)$ \\
\hline \multicolumn{7}{|c|}{ Worry/fear of being infected with COVID-19 (Likert score, 1-5) } \\
\hline Mean \pm SD & $3.63 \pm 1.09$ & $3.75 \pm 1.05$ & $3.52 \pm 1.04$ & $3.02 \pm 1.10$ & $2.76 \pm 1.01$ & $2.35 \pm 1.10$ \\
\hline Median (Min, Max) & $4(1,5)$ & $4.00(1,5)$ & $4.00(1,5)$ & $3.00(1,5)$ & $3(1,5)$ & $2.00(1,5)$ \\
\hline \multicolumn{7}{|c|}{ Knowledge about COVID-19 (Yes): $n(\%)$} \\
\hline $\begin{array}{l}\text { Possibility of being re-infected after } \\
\text { recovering from COVID-19 }\end{array}$ & $5514(83.9)$ & $4180(85.9)$ & $956(76.8)$ & $98(80.3)$ & $177(88.9)$ & $103(74.6)$ \\
\hline $\begin{array}{l}\text { COVID-19 can be prevented by } \\
\text { vaccination }\end{array}$ & $5407(82.3)$ & $4428(91.0)$ & $715(57.4)$ & $78(63.9)$ & $108(54.3)$ & $78(56.5)$ \\
\hline $\begin{array}{l}\text { There is currently an effective vaccine } \\
\text { against COVID-19 }\end{array}$ & $4361(66.4)$ & $3738(76.8)$ & $421(33.8)$ & $80(65.6)$ & $88(44.2)$ & $34(24.6)$ \\
\hline \multicolumn{7}{|c|}{ Knowledge about COVID-19 (composite score, 0-3) * } \\
\hline Mean \pm SD & $2.33 \pm 0.88$ & $2.54 \pm 0.75$ & $1.68 \pm 0.93$ & $2.10 \pm 0.86$ & $1.87 \pm 0.90$ & $1.56 \pm 0.94$ \\
\hline Median (Min, Max) & $3(0,3)$ & $3(0,3)$ & $2(0,3)$ & $2(0,3)$ & $2(0,3)$ & $1.5(0,3)$ \\
\hline \multicolumn{7}{|c|}{ Importance of COVID-19 vaccination to protect others (Likert score, $1-5$ ) } \\
\hline Mean \pm SD & $4.87 \pm 0.85$ & $4.76 \pm 0.65$ & $4.09 \pm 1.04$ & $3.75 \pm 1.00$ & $4.17 \pm 0.88$ & $3.36 \pm 1.49$ \\
\hline Median (Min, Max) & $5(1,5)$ & $5(1,5)$ & $4(1,5)$ & $4(1,5)$ & $4(1,5)$ & $4(1,5)$ \\
\hline Not at all important & $158(2.4)$ & $73(1.5)$ & $45(3.6)$ & $6(4.9)$ & $3(1.5)$ & $31(22.5)$ \\
\hline A little important & $97(1.5)$ & $28(0.6)$ & $57(4.6)$ & $1(0.8)$ & $3(1.5)$ & $8(5.8)$ \\
\hline Moderately important & $326(5.0)$ & $73(1.5)$ & $177(14.2)$ & $26(21.3)$ & $36(18.1)$ & $14(10.1)$ \\
\hline Very important & $1258(19.1)$ & $651(13.4)$ & $433(34.8)$ & $51(41.8)$ & $73(36.7)$ & $50(36.2)$ \\
\hline Extremely important & $4732(72.0)$ & $4042(83.0)$ & $533(42.8)$ & $38(31.1)$ & $84(42.2)$ & $35(25.4)$ \\
\hline
\end{tabular}

Note. Percentages (\%) are within country comparisons. ${ }^{+}$Countries in Africa in this study comprised of DR Congo $(n=117)$, Uganda $(n=8)$, and Malawi $(n=13) .{ }^{*}$ Reference Group.

At $50 \%$ and $75 \%$ effectiveness, individuals aged 30-39 years old were least likely to accept vaccination of children but were most likely to accept the vaccination in cases of an effectiveness level of $95 \%$. Females were more in favor of child vaccination than males at $75 \%$ effectiveness (aOR: 1.17, 95\%CI [1.01, 1.35], $p=0.032$ ). However, at $95 \%$ effectiveness, females were less in favor of child vaccination compared to males (aOR: 0.54, 95\% CI [0.44, $0.73], p<0.001)$. At $50 \%$ vaccine effectiveness, individuals who lived with children 12 to 17 years old had higher acceptance rates for the vaccination of children (aOR: 1.27, 95\% CI [1.07, 1.50], $p=0.006$ ), while at $95 \%$ effectiveness level, those who lived with children $<12$ years old were more likely to accept child vaccination (aOR: 1.30, 95\%CI [1.04, 1.63], $p=0.023$ ). Those with an undergraduate degree were more willing to vaccinate children compared to individuals with primary/secondary education, with the highest odds ratio at $95 \%$ effectiveness (aOR: 2.33, 95\%CI [1.71, 3.19], $p<0.001$ ).

At $95 \%$ effectiveness, individuals with upper-middle income had higher odds of accepting vaccination for children (aOR: 1.77, 95\% CI [1.04, 3.00], $p=0.034)$ whilst those living in urban area (aOR: $0.47,95 \% \mathrm{CI}[0.28,0.79], p=0.0 .5)$ had lower odds of vaccine acceptance for children.

At $50 \%$ and $75 \%$ effectiveness levels, participants who had tested positive for COVID19 were less likely to accept vaccination of children (aOR: 0.78, 95\%CI [0.61, 0.99], $p=0.043$ and aOR: $0.76,95 \% \mathrm{CI}[0.60,0.97], p=0.027$, respectively).

Across all effectiveness levels, individuals who were more worried/fearful about COVID-19, who had higher knowledge about COVID-19, and who believed that taking the COVID-19 vaccine is important to protect others, were more likely to accept COVID-19 vaccination of children (Table 4). 
Table 3. Bivariate analysis of associations between demographic variables, knowledge, and attitudes towards COVID-19 vaccination and vaccine effectiveness.

\begin{tabular}{|c|c|c|c|c|c|c|c|c|c|c|c|c|}
\hline \multirow{2}{*}{ Variable } & \multicolumn{4}{|c|}{$50 \%$ Effectiveness } & \multicolumn{4}{|c|}{$75 \%$ Effectiveness } & \multicolumn{4}{|c|}{ 95\% Effectiveness } \\
\hline & $n(\%)$ & $x^{2}$ & $p$ & Effect Size * & $n(\%)$ & $x^{2}$ & $p$ & Effect Size * & $n(\%)$ & $x^{2}$ & $p$ & Effect Size * \\
\hline Age (Years) & & 152.96 & $<0.001$ & 0.163 & & 138.97 & $<0.001$ & 0.151 & & 18.21 & 0.001 & 0.053 \\
\hline $18-29$ & $401(50.5)$ & & & & 507 (59.9) & & & & $859(90.9)$ & & & \\
\hline $30-39$ & $656(57.4)$ & & & & $827(68.1)$ & & & & $1226(92.9)$ & & & \\
\hline $40-49$ & $683(62.6)$ & & & & $841(71.8)$ & & & & $1148(91.5)$ & & & \\
\hline $50-59$ & $925(68.8)$ & & & & $1078(76.4)$ & & & & $1402(93.6)$ & & & \\
\hline 60 and above & $1026(73.4)$ & & & & $1179(80.6)$ & & & & $1472(94.7)$ & & & \\
\hline Country & & 1277.05 & $<0.001$ & 0.470 & & 1545.75 & $<0.001$ & 0.503 & & 379.54 & $<0.001$ & 0.240 \\
\hline Malaysia & $289(27.2)$ & & & & $383(35.2)$ & & & & $1060(85.1)$ & & & \\
\hline Thailand & $15(13.0)$ & & & & $23(20.2)$ & & & & $109(89.3)$ & & & \\
\hline Bangladesh & $59(34.1)$ & & & & $93(50.0)$ & & & & $185(93.0)$ & & & \\
\hline African Countries ${ }^{\dagger}$ & $15(12.4)$ & & & & $16(13.4)$ & & & & $86(62.3)$ & & & \\
\hline Gender & & 12.43 & $<0.001$ & 0.046 & & 20.58 & $<0.001$ & 0.058 & & 19.46 & $<0.001$ & 0.054 \\
\hline Male & $1264(61.0)$ & & & & $1509(69.1)$ & & & & $2105(91.0)$ & & & \\
\hline Female & $2427(65.6)$ & & & & $2923(74.5)$ & & & & $4002(94.0)$ & & & \\
\hline Education Level & & 109.43 & $<0.001$ & 0.138 & & 137.42 & $<0.001$ & 0.150 & & 61.39 & $<0.001$ & 0.097 \\
\hline Primary/Secondary & $398(55.4)$ & & & & $486(64.3)$ & & & & $748(88.9)$ & & & \\
\hline Postgraduate & $2073(70.4)$ & & & & $2480(79.1)$ & & & & $3150(95.3)$ & & & \\
\hline Household & & & & & & & & & & & & \\
\hline Living with children $<12$ years old & & 47.68 & $<0.001$ & 0.091 & & 50.58 & $<0.001$ & 0.091 & & 11.55 & 0.001 & 0.042 \\
\hline Yes & $989(57.3)$ & & & & $1210(66.4)$ & & & & $1830(91.3)$ & & & \\
\hline No & $2702(66.8)$ & & & & $3222(75.2)$ & & & & $4277(93.7)$ & & & \\
\hline $\begin{array}{c}\text { Living with children } 12 \text { to } 17 \\
\text { years old }\end{array}$ & & 22.76 & $<0.001$ & 0.063 & & 30.14 & $<0.001$ & 0.070 & & 13.36 & $<0.001$ & 0.045 \\
\hline Yes & $678(58.0)$ & & & & $819(66.4)$ & & & & $1224(90.7)$ & & & \\
\hline No & $3013(65.5)$ & & & & $3613(74.2)$ & & & & $4883(93.5)$ & & & \\
\hline Socio-economic status & & 49.68 & $<0.001$ & 0.093 & & 80.18 & $<0.001$ & 0.115 & & 16.22 & $<0.001$ & 0.050 \\
\hline Low/Lower Middle & $1669(59.9)$ & & & & $1971(67.4)$ & & & & $2911(91.6)$ & & & \\
\hline Upper Middle & $1770(66.7)$ & & & & $2168(76.7)$ & & & & $2850(94.2)$ & & & \\
\hline High & $252(76.1)$ & & & & $293(82.3)$ & & & & $346(93.8)$ & & & \\
\hline Residential setting & & 76.99 & $<0.001$ & 0.116 & & 88.80 & $<0.001$ & 0.121 & & 3.49 & 0.175 & 0.023 \\
\hline Suburban/Urban Slum & $433(62.1)$ & & & & $515(70.0)$ & & & & $726(92.4)$ & & & \\
\hline Urban & $3166(65.5)$ & & & & $3796(74.2)$ & & & & $5115(93.2)$ & & & \\
\hline Rural & $92(38.0)$ & & & & $121(47.6)$ & & & & $266(90.5)$ & & & \\
\hline
\end{tabular}


Table 3. Cont.

\begin{tabular}{|c|c|c|c|c|c|c|c|c|c|c|c|c|}
\hline \multirow{2}{*}{ Variable } & \multicolumn{4}{|c|}{$50 \%$ Effectiveness } & \multicolumn{4}{|c|}{$75 \%$ Effectiveness } & \multicolumn{4}{|c|}{ 95\% Effectiveness } \\
\hline & $n(\%)$ & $x^{2}$ & $p$ & Effect Size * & $n(\%)$ & $x^{2}$ & $p$ & Effect Size * & $n(\%)$ & $x^{2}$ & $p$ & Effect Size * \\
\hline Healthcare worker or student & & 5.65 & 0.017 & 0.031 & & 3.61 & 0.057 & 0.024 & & 0.39 & 0.530 & 0.008 \\
\hline Yes & $1076(61.7)$ & & & & $1296(70.9)$ & & & & $1835(93.2)$ & & & \\
\hline No & $2615(65.0)$ & & & & $3136(73.3)$ & & & & $4272(92.8)$ & & & \\
\hline Working/studying from home & & 24.18 & $<0.001$ & 0.065 & & 45.53 & $<0.001$ & 0.086 & & 19.15 & $<0.001$ & 0.054 \\
\hline Yes & $2221(66.6)$ & & & & $2709(75.8)$ & & & & $3609(94.1)$ & & & \\
\hline No & $1470(60.3)$ & & & & $1723(68.0)$ & & & & 2498 (91.3) & & & \\
\hline COVID-19 Testing & & 20.79 & $<0.001$ & 0.060 & & 21.73 & $<0.001$ & 0.060 & & 8.03 & 0.018 & 0.035 \\
\hline Tested Positive & $364(70.3)$ & & & & $431(78.6)$ & & & & $540(93.4)$ & & & \\
\hline $\begin{array}{c}\text { Not Tested/ Does not know test } \\
\text { results }\end{array}$ & 1945 (61.6) & & & & $2356(70.4)$ & & & & $3330(92.1)$ & & & \\
\hline & Mean (SD) & $t$ value & $p$ & Effect size $\S$ & Mean (SD) & $t$ value & $p$ & Effect size $\S$ & Mean (SD) & $t$ value & $p$ & Effect size $\S$ \\
\hline $\begin{array}{l}\text { Worry/fear of being infected with } \\
\text { COVID-19 }\end{array}$ & $3.86(0.98)$ & 20.13 & $<0.001$ & 0.694 & $3.82(0.98)$ & 19.29 & $<0.001$ & 0.766 & $3.71(1.03)$ & 16.65 & $<0.001$ & 1.479 \\
\hline Knowledge about COVID-19 $\ddagger$ & $2.65(0.62)$ & 35.88 & $<0.001$ & 1.308 & $2.63(0.63)$ & 39.73 & $<0.001$ & 1.678 & $2.41(0.81)$ & 30.57 & $<0.001$ & 0.754 \\
\hline $\begin{array}{l}\text { Importance of vaccination to } \\
\text { protect others }\end{array}$ & $4.84(0.43)$ & 28.03 & $<0.001$ & 1.148 & $4.82(0.44)$ & 28.93 & $<0.001$ & 1.352 & $4.69(0.61)$ & 24.78 & $<0.001$ & 2.276 \\
\hline
\end{tabular}

Note. ${ }^{\dagger}$ Countries in Africa in this study comprised of DR Congo $(n=117)$, Uganda $(n=8)$, and Malawi $(n=13)$. $\ddagger$ Knowledge was the sum of answering correctly $(=1$ point each) to the following questions: (1) possibility of being re-infected after recovering from a previous COVID-19 infection; (2) COVID-19 infection could be prevented by a vaccine; and (3) there is currently an effective vaccine against COVID-19. * Cramer's V. § Cohen's d.

Table 4. Multiple logistic regression models of factors predicting vaccine acceptance for child at 50\%, $75 \%$, and $95 \%$ effectiveness levels.

\begin{tabular}{|c|c|c|c|c|c|c|c|c|c|c|c|c|}
\hline \multirow{3}{*}{ Variables } & \multicolumn{4}{|c|}{$50 \%$ Effectiveness ${ }^{a}$} & \multicolumn{4}{|c|}{$75 \%$ Effectiveness ${ }^{b}$} & \multicolumn{4}{|c|}{$95 \%$ Effectiveness $^{c}$} \\
\hline & \multirow{2}{*}{$\mathrm{aOR}$} & \multicolumn{2}{|c|}{$95 \% \mathrm{CI}$} & \multirow{2}{*}{$p$-Value } & \multirow{2}{*}{ aOR } & \multicolumn{2}{|c|}{$95 \% \mathrm{CI}$} & \multirow{2}{*}{$p$-Value } & \multirow{2}{*}{ aOR } & \multicolumn{2}{|c|}{$95 \%$ CI } & \multirow{2}{*}{$p$-Value } \\
\hline & & Lower & Upper & & & Lower & Upper & & & Lower & Upper & \\
\hline $\begin{array}{c}\text { Constant } \\
\text { Age (Years) }\end{array}$ & 0.05 & & & & 0.09 & & & & 0.05 & & & \\
\hline $18-29$ & 0.62 & 0.46 & 0.82 & 0.001 & 0.63 & 0.46 & 0.86 & 0.003 & 0.98 & 0.64 & 1.49 & 0.922 \\
\hline $30-39$ & 0.47 & 0.36 & 0.62 & $<0.001$ & 0.47 & 0.35 & 0.63 & $<0.001$ & 2.00 & 1.31 & 3.05 & 0.001 \\
\hline $40-49$ & 0.85 & 0.64 & 1.13 & 0.253 & 0.73 & 0.53 & 0.99 & 0.040 & 1.17 & 0.77 & 1.78 & 0.466 \\
\hline
\end{tabular}


Table 4. Cont.

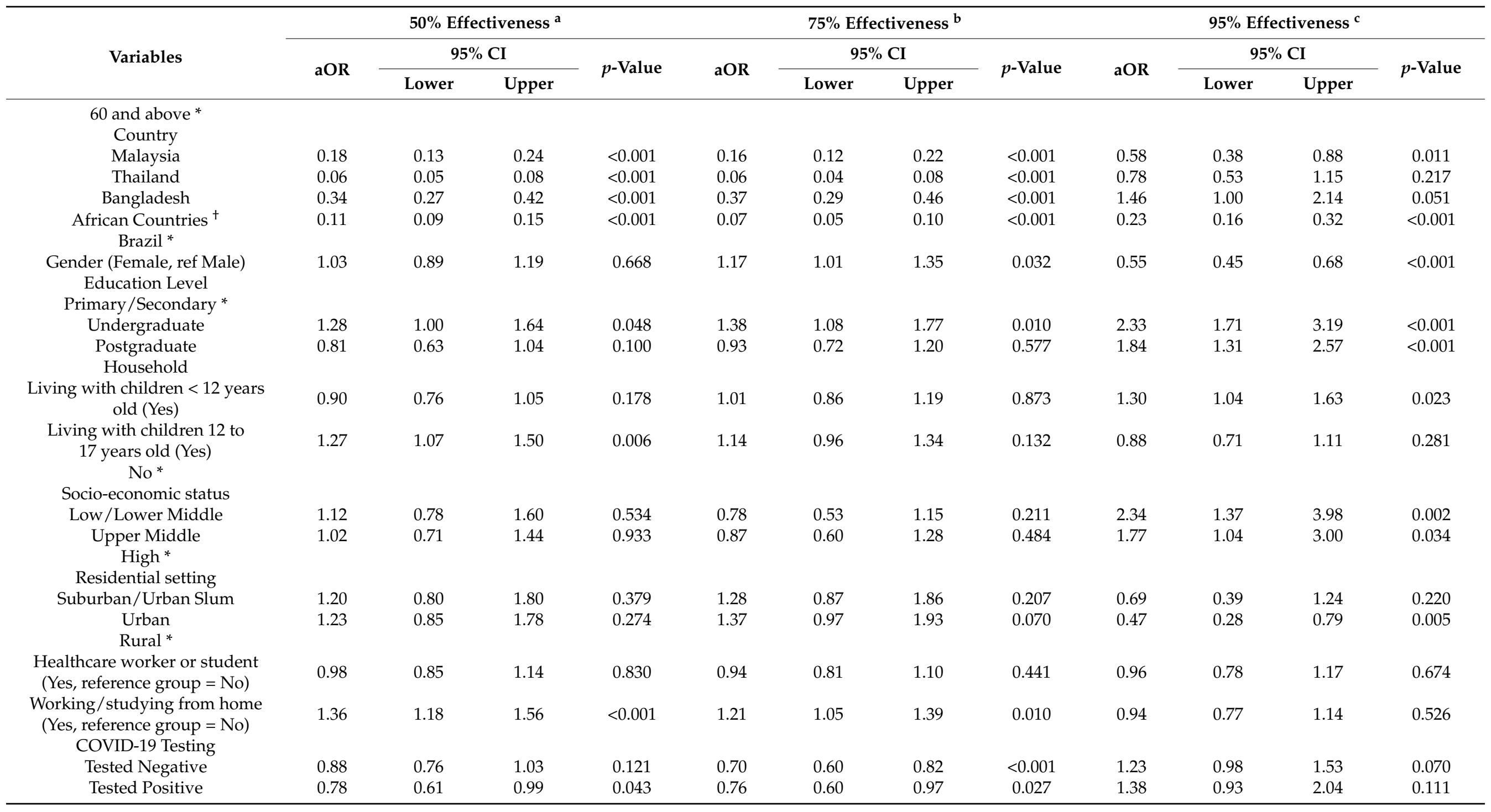


Table 4. Cont.

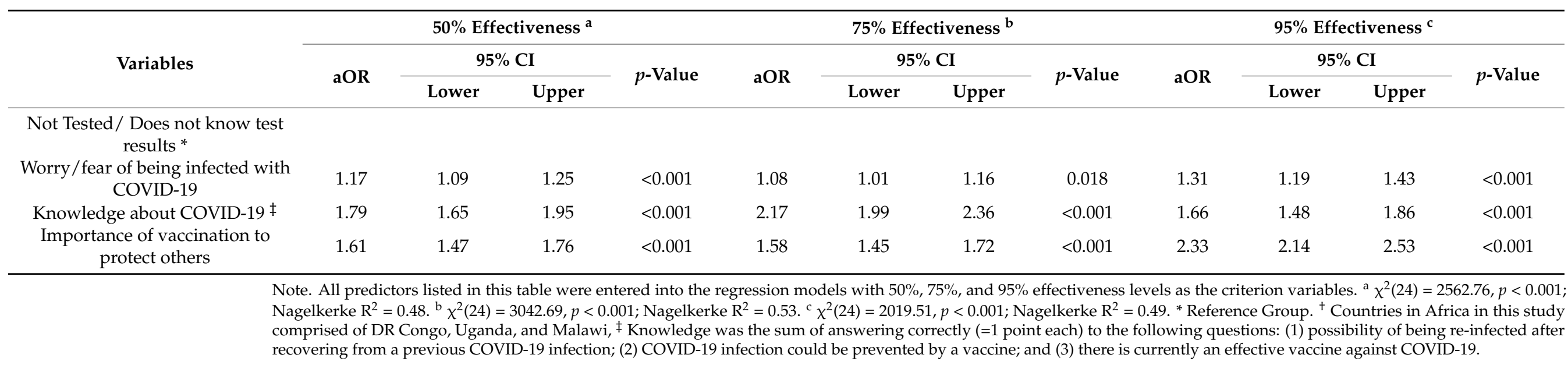




\section{Discussion}

With the increasing availability of COVID-19 vaccines worldwide, understanding the factors influencing parents' or caregivers' willingness to vaccinate their children is critically important for vaccine policy decisions. In this international online survey $92.9 \%$ of the participants were willing to vaccinate children at $95 \%$ vaccine effectiveness, which is high in comparison with other studies. In an international cross-sectional survey of emergency departments in six countries, $65 \%$ of caregivers reported that they intended to vaccinate their child against COVID-19, once a vaccine was available [20]. In a study among factory workers in Shenzhen, China, $72.6 \%$ of parents would accept COVID-19 vaccination of their children [31]. In Zambia, in a nested study within a measles-rubella mass vaccination campaign, $92 \%$ of the parents reported that they intended to have their child vaccinated against COVID-19 [19]. Further, in the UK, an online survey found that $48.2 \%$ of parents or guardians would accept COVID-19 vaccination of their children aged 18 months or under [32]. However, in another study conducted in a children's hospital in Ankara, Turkey, $28.9 \%$ of the parents reported that they would not allow their child to be vaccinated with foreign COVID-19 vaccines, while $56.8 \%$ said they would if the vaccine was a domestic vaccine [33].

In our survey, the willingness to vaccinate a child for COVID-19 varied between countries and was also found to increase as the vaccine effectiveness level increased. Other studies also showed that the strongest predictor of intent to vaccinate children was vaccine safety and efficacy [19,34-37]. In a study in the United States, less than one-half of the parents reported that they were likely to have their child receive a COVID-19 vaccine mainly because of safety concerns [38].

The highest vaccination acceptance was noted among the Brazilian participants (95.5\%). African and Malaysian participants were less likely to have children vaccinated. It is worth noting that the participants from these countries were significantly younger than Brazilian participants (analysis not shown) and therefore may have had younger children than the other countries. Moreover, at the time of this survey, in Malaysia and Africa, there was a lot of misinformation with regards to the COVID-19 vaccine safety and its efficacy $[39,40]$. For instance, it was believed that mixed messages coming from political figures created unjustified fears against the adverse effects of H1N1 vaccination [41]. On the other hand, Brazil had the second highest number of COVID-19-related deaths in the world in early 2021 [42]. This situation may have increased the willingness to vaccinate children as the benefits of inoculating against COVID-19 may have been seen as outweighing the potential adverse events.

In our study, male participants were more willing to vaccinate children at the $95 \%$ effectiveness level, and this was also seen in a survey in high-income countries [43]. A study on H1N1 vaccination also showed similar results, where fathers were more willing than mothers to vaccinate their child [34]. An explanation could be that males may differ from females in decision-making behavior due to their higher likelihood to take risks. Future in-depth studies are needed to examine these gender dynamics.

Participants from urban areas had 53\% lower odds of accepting the vaccine for their children at the $95 \%$ effectiveness level. Similarly, past studies also found that caregivers from urban sites were less likely to vaccinate their children than those from rural sites [44]. These results are of concern as the prevalence of COVID-19 positive cases in urban areas has been shown to be higher than in rural areas and small towns [45,46]. Our findings could be due to the greater exposure in urban areas to information from the internet and social media. Online misinformation has been found to correlate with vaccine hesitancy and thus lower the vaccination coverage [19,47-49]. Thus, more trusted and reliable sources of information ought to be conveyed to the community, to increase their confidence in the vaccine.

Educational level was strongly associated with willingness to vaccinate children. Undergraduates were more in favor of vaccinating children at all effectiveness levels. Educational level is closely related to knowledge about COVID-19 vaccines. At all three 
levels of vaccine effectiveness, knowledge about COVID-19 vaccines was associated with willingness to vaccinate a child. So, higher exposure to correct information about COVID19 benefits vaccine uptake. Knowledge of the disease and understanding that vaccines are effective prevention strategies were also associated with increased H1N1 vaccination uptake [47]. Similar to our findings, parents with post-secondary education have been shown to be more willing to vaccinate their children [44,50,51]. Studies have also shown that children of parents with a higher educational level were more likely to be vaccinated [34,51]. As non-compliance with childhood vaccination has increased in the past ten years [52,53] it is important that decisions to introduce vaccination of children with a new vaccine are taken with sufficient knowledge about the efficacy and potential adverse effects of these vaccines and consider the acceptance of these vaccines by the public, particularly the parents.

Income was also shown to be associated with willingness to vaccinate children against COVID-19. Those with low, lower middle, and upper middle-income status had higher odds of accepting child vaccination at the $95 \%$ vaccine effectiveness level, compared to those in the high-income group. These findings contrast with other studies undertaken in the US, where there was a lower level of vaccine acceptance among lower-income groups [54,55]. The COVID-19 pandemic has particularly impacted those belonging to lower socio-economic status groups and thus it is important to prevent further inequity in COVID-19 vaccine distribution and uptake, particularly in disadvantaged communities. Due to the environmental and sanitary conditions that these communities are living in, such as overcrowding, members are more prone to contracting the disease and more likely to die from it, thus, one would expect them to accept the COVID-19 vaccination, not only for their children, but also for themselves.

Participants who tested positive for COVID-19 had 24\% lower odds for being prepared to have their children vaccinated at the $50 \%$ and $75 \%$ vaccine effectiveness levels. The reason for this may be that with such a low level of effectiveness, it may not be viewed as beneficial to expose children to vaccines for which there is limited experience concerning potential side effects in children [56]. Conversely, a previous study found no correlation between family members diagnosed with COVID-19 and their willingness to vaccinate their child [57].

Another factor that may affect the acceptance of the vaccine for children could be living with children, as this may increase the risk of contracting the virus if the children are virus carriers [25]. In our study, those working or studying from home were more open to the vaccination of children. The explanation could be that, for them, contact with children could be their main risk for becoming infected.

Worry about becoming infected with COVID-19 was also significantly associated with vaccine acceptance for children. This concurs with other findings elsewhere. A study found that $92 \%$ of caregivers were worried about getting the disease or their family being infected and, among these, 93\% would vaccinate their children [31]. Related to the HINI vaccination, there was a strong association between vaccine intentions and fear of the adult and child catching the disease [19], while concern about the COVID-19 outbreak in Australia was associated with enhanced willingness to get vaccinated [58]. According to the health belief model, those who were more worried/fearful of COVID-19 may be more likely to seek relief for their adverse emotional condition by accepting the vaccine, including for children [59]. Therefore, fear or worry could be a catalyst for the vaccination of children and to achieve herd immunity. For the uptake of health behaviors, an understanding of these factors affecting people's intentions for vaccination is deemed important for the success of any vaccination program. The factors highlighted in this study will further assist policy makers to plan and develop strategies for their target groups for vaccinations, based on socio-demographic data.

In our study, those who believed that vaccination was important to protect others had 2.33 times, 1.58 times and 1.61 times higher odds of accepting having children vaccinated at $95 \%, 75 \%$, and 50\% vaccine effectiveness, respectively. Similarly, a study found that the main reasons for vaccine acceptance was self-protection (42\%) and protecting the child 
(42\%) from COVID-19, with 48.2\% leaning towards accepting a COVID-19 vaccine for their child [32]. Goldman and colleagues reported that the most common reason given by caregivers intending to vaccinate their children was to protect their children (62\%) [20]. Similar to our findings, Goldman and colleagues reported that "protection of others" was the second common theme given by their willing caregivers. The low prevalence of acceptance could be because children have a lower susceptibility to develop severe COVID-19 disease and their role in the transmission of COVID-19 is unclear [60,61].

A recent review about the reasons for vaccine hesitancy in LMICs showed that exposure to misinformation about COVID-19 vaccines, public concerns over the safety of vaccines, and distrust in the government, were the main contributing factors to low vaccine acceptance rates [18]. Therefore, to decrease vaccine hesitancy, direct engagement with communities through influencers, including community leaders and health experts, is needed. Moreover, clear and transparent communication, with strong endorsement by health care workers, is very important [62]. Thus, clear and consistent communication and accurate information is important in helping people decide whether they want to be vaccinated or not. Further, whether it is vaccines for adults or children, policy makers, government officials, and the media should pay attention to the spread of data which is not supported by scientific evidence that may affect vaccine acceptance [62].

Our study is not without limitations. Our method of using online social media and online communicators, such as WhatsApp and Facebook, to distribute the questionnaire could lead to biased results. This is because individuals belonging to low-income countries may have limited internet access, and therefore the responses may originate from individuals with higher income levels who could afford an electronic device or internet connection to access this questionnaire. Moreover, it may also limit the participants to those within the researchers' personal and professional networks, and this creates a non-representative, biased study population. Future studies should consider conducting surveys selecting participants randomly via telephone or a paper questionnaire to ensure a more representative study population. However, such surveys are more difficult to organize, are not feasible in certain settings (telephone numbers may not be available) and are costly. Due to the COVID-19 pandemic, we have chosen the online survey method to obtain rapid information at low cost as a basis for designing more in-depth studies when the COVID-19 vaccines will be more widely available for children. We considered the household composition of the participants, but we did not specifically ask parents whether they would be willing to vaccinate their own children. We also did not specify the child's age at which they would accept vaccination - the decision to vaccinate a young child may be seen as being riskier than to vaccinate a near-adult child. Other influencing factors also need to be considered, such as attitudes towards all vaccinations, and social media influence which can affect ones' perception of vaccine acceptability. The validity, reliability, and intelligibility of the questionnaire was not thoroughly assessed in the target population. We did not specify in the questionnaire the meaning of the term "effectiveness of the COVID-19 vaccine"; this could be effectiveness against infection, severe COVID-19, hospitalization, or death. It seems most probable that most respondents interpreted the question as effectiveness against infection. As in any cross-sectional study design, causal inference cannot be made. It is also important to mention that this survey was carried out prior to the COVID-19 vaccination roll-out in the surveyed countries in this study. So, over time, perceptions about COVID-19 vaccination may have changed. For example, studies investigating the safety, immunogenicity, and efficacy of the COVID-19 vaccine in children [63] may change parents' and caregivers' willingness to vaccinate their children.

In November 2021, the US Centers for Disease Control and Prevention recommended Pfizer's COVID-19 mRNA vaccine for children between 5 and 11 years [64]. Yet surveys in the United States showed that $42 \%$ to $66 \%$ of parents were reluctant or opposed to vaccinate their children [64]. Without vaccination, it is likely that almost everyone, including young children, will be COVID-19 infected at some point in their lives [65]. So, the question is: which is worse for children, vaccination or natural infection? In high-income countries, 
vaccination of children has started on a large scale and vaccine acceptance rates have also changed over time [65]. The successful vaccination program in Israel, and to a lesser degree in the United States, led to increased willingness by parents to vaccinate their children younger than 12 years against COVID-19. In Canada, a low rate of vaccination of adults was associated with lower willingness to vaccinate children [65]. A follow-up study should also be carried in LMICs once vaccination for children against COVID-19 has become more widely available.

\section{Conclusions}

More than half the respondents were willing to vaccinate children. Factors influencing willingness to vaccinate children were parental gender, age, education, income level, residential setting, knowledge, worry about being infected, and the importance of vaccination to protect self or others. Findings from this study are useful for policy decisions about COVID-19 vaccination of children. Vaccination may reduce the spread of infection in schools and nurseries. However, according to current evidence, children rarely develop severe symptoms due to COVID-19; therefore, administering the COVID-19 vaccine in children may be of limited or no benefit for the health of the children. Alternatively, vaccinating school children may decrease COVID-19 transmission in school and in this way may avoid school closures. Keeping schools open is very important for the education and development of the children. Another reason to vaccinate children, still being assessed, is to prevent them transmitting the infection to persons at risk of severe disease, such as elderly people and persons with underlying chronic diseases. However, given that the latter are increasingly being protected by vaccination, and other public health interventions, the need to vaccinate children, and at what age, are still very much under debate. Within this debate, the opinion of the children and their parents needs to be considered to design appropriate policies regarding vaccination of children against COVID-19.

Author Contributions: Conceptualization, S.A.B., E.F.d.M.V., S.P., J.N.S.F., W.Y.L. and R.C.; methodology, S.A.B., E.F.d.M.V., C.S.S., W.S.C., S.P., M.T.H., P.S., J.D.D., B.O.A., M.C.H., H.D., J.N.S.F., W.Y.L. and R.C.; software, W.S.C.; formal analysis, C.S.S. and W.S.C.; data curation. S.A.B., C.S.S., W.S.C. and W.Y.L.; writing-original draft preparation, S.A.B., C.S.S., W.S.C., W.Y.L. and R.C.; writing-all authors reviewed and edited the manuscript; supervision, S.A.B., E.F.d.M.V., W.Y.L. and R.C.; project administration, S.A.B., E.F.d.M.V., S.P., W.Y.L. and R.C.; funding acquisition, R.C. All authors have read and agreed to the published version of the manuscript.

Funding: This research was funded by VLIRUOS (Flemish Interuniversity Council for University Development Cooperation).

Institutional Review Board Statement: The study was conducted according to the guidelines of the Declaration of Helsinki, and was approved by the institutional review boards in the following countries: Brazil: National Research Ethics Commission, CAAE: 30343820.9.0000.0008, report number 4.518.164; Malaysia: Universiti Malaya Research Ethics Committee, protocol code UM.TNC2/UMREC_1165, 27 January 2021; Thailand: The Committee for Research Ethics (Social Sciences), Mahidol University, ethics code 2020/127 (B1); Bangladesh: Biosafety, Biosecurity \& Ethical Committee, Faculty of Biological Sciences, Jahangirnagar University, Savar, Dhaka, Bangladesh, ethics code: BBEC, JU / M-2020 (6)I/ RG/ Bangladesh; DRC: National Ethical Committee, reference number CNES No. 75/CNES/BN/PMMF/2020; Uganda: The Ethics Committee of School of Public health, Makerere University, Kampala, Uganda, HDREC number 809; Malawi: University of North Carolina Institutional Review Board and the Malawi National Health Sciences Research Committee reviewed the protocol and provided an exemption as "human subjects research" given the online survey nature of the work; Belgium: The Ethics Committee of the University Hospital Antwerp 20/13/148.

Informed Consent Statement: Informed consent was obtained from all participants involved in the study.

Data Availability Statement: Data are available upon reasonable request. Data are available on the International Consortium (International Citizen Project COVID-19 (ICPcovid): http:/ /www.icpcovid. 
com, accessed on 4 November 2021) website and can be used by other investigators on request. De-identified participant data are available.

Acknowledgments: We would like to thank all our participants. We would also like to acknowledge Asma Binte Aziz, Sultan Mahmood, and Syeda Fatema Alam (Bangladesh) for their role in data collection.

Conflicts of Interest: The authors declare no conflict of interest.

\section{References}

1. World Health Organization. Rolling Updates on Coronavirus Disease (COVID-19). Available online: https:/ /www.who.int/ emergencies/diseases/novel-coronavirus-2019/events-as-they-happen (accessed on 2 September 2020).

2. World Health Organization. WHO Coronavirus (COVID-19) Dashboard. Available online: https://covid19.who.int/table (accessed on 4 November 2021).

3. Centers for Disease Control and Prevention. COVID-19 Vaccine Emergency Use Authorization (EUA) Fact Sheets for Recipients and Caregivers. Available online: https:/ / www.cdc.gov/vaccines/covid-19/eua/index.html (accessed on 17 September 2021).

4. World Health Organization. Statement for Healthcare Professionals: How COVID-19 Vaccines Are Regulated for Safety and Effectiveness. Available online: https://www.who.int/news/item/11-06-2021-statement-for-healthcare-professionals-howcovid-19-vaccines-are-regulated-for-safety-and-effectiveness (accessed on 4 November 2021).

5. Zhu, F.-C.; Guan, X.-H.; Li, Y.-H.; Huang, J.-Y.; Jiang, T.; Hou, L.-H.; Li, J.-X.; Yang, B.-F.; Wang, L.; Wang, W.-J.; et al. Immunogenicity and safety of a recombinant adenovirus type-5-vectored COVID-19 vaccine in healthy adults aged 18 years or older: A randomised, double-blind, placebo-controlled, phase 2 trial. Lancet 2020, 396, 479-488. [CrossRef]

6. Folegatti, P.M.; Ewer, K.J.; Aley, P.K.; Angus, B.; Becker, S.; Belij-Rammerstorfer, S.; Bellamy, D.; Bibi, S.; Bittaye, M.; Clutterbuck, E.A.; et al. Safety and immunogenicity of the ChAdOx1 nCoV-19 vaccine against SARS-CoV-2: A preliminary report of a phase 1/2, single-blind, randomised controlled trial. Lancet 2020, 396, 467-478. [CrossRef]

7. European Medicines Agency. EMA Recommends First COVID-19 Vaccine for Authorisation in the EU. Available online: https: //www.ema.europa.eu/en/news/ema-recommends-first-covid-19-vaccine-authorisation-eu (accessed on 23 September 2021).

8. Our World in Data. People Fully Vaccinated per Hundred. Available online: https://ourworldindata.org/grapher/share-peoplefully-vaccinated-covid?tab=table (accessed on 4 November 2021).

9. Centers for Disease Control and Prevention. COVID-19 Vaccines for Children and Teens. Available online: https:/ /www.cdc. gov/coronavirus/2019-ncov/vaccines/recommendations/children-teens.html (accessed on 4 November 2021).

10. Government of Canada. Health Canada Authorizes Use of the Pfizer-BioNTech COVID-19 Vaccine in Children 12 to 15 Years of Age. Available online: https:/ /www.canada.ca/en/health-canada/news/2021/05/health-canada-authorizes-use-of-thepfizer-biontech-covid-19-vaccine-in-children-12-to-15-years-of-age.html (accessed on 4 November 2021).

11. Singh, L.; Bansal, S.; Bode, L.; Budak, C.; Chi, G.; Kawintiranon, K.; Padden, C.; Vanarsdall, R.; Vraga, E.; Wang, Y. A first look at COVID-19 information and misinformation sharing on Twitter. arXiv 2003, arXiv:2003.13907.

12. World Health Organization. Ten Threats to Global Health in 2019. 2020. Available online: https://www.who.int/news-room/ spotlight/ten-threats-to-global-health-in2019 (accessed on 4 November 2021).

13. Cornwall, W. Just 50\% of Americans Plan to Get a COVID-19 Vaccine. Here's How to Win Over the Rest. Available online: https: / /www.sciencemag.org/news/2020/06/just-50-americans-plan-get-covid-19-vaccine-here-s-how-win-over-rest (accessed on 4 November 2021).

14. Lazarus, J.V.; Ratzan, S.C.; Palayew, A.; Gostin, L.O.; Larson, H.J.; Rabin, K.; Kimball, S.; El-Mohandes, A. A global survey of potential acceptance of a COVID-19 vaccine. Nat. Med. 2021, 27, 225-228. [CrossRef]

15. Chirumbolo, S. Vaccination hesitancy and the "myth" on mRNA-based vaccines in Italy in the COVID-19 era: Does urgency meet major safety criteria? J. Med Virol. 2021, 93, 4049-4053. [CrossRef]

16. Zimmermann, P.; Curtis, N. Why is COVID-19 less severe in children? A review of the proposed mechanisms underlying the age-related difference in severity of SARS-CoV-2 infections. Arch. Dis. Child. 2021, 106, 429-439. [CrossRef]

17. Principi, N.; Esposito, S. Why It Is Important to Develop an Effective and Safe Pediatric COVID-19 Vaccine. Vaccines 2021, 9, 127. [CrossRef]

18. Moola, S.; Gudi, N.; Nambiar, D.; Dumka, N.; Ahmed, T.; Sonawane, I.R.; Kotwal, A. A rapid review of evidence on the determinants of and strategies for COVID-19 vaccine acceptance in low- and middle-income countries. J. Glob. Health 2021, 11, 11. [CrossRef]

19. Carcelen, A.C.; Prosperi, C.; Mutembo, S.; Chongwe, G.; Mwansa, F.D.; Ndubani, P.; Simulundu, E.; Chilumba, I.; Musukwa, G.; Thuma, P.; et al. COVID-19 vaccine hesitancy in Zambia: A glimpse at the possible challenges ahead for COVID-19 vaccination rollout in sub-Saharan Africa. Hum. Vaccines Immunother. 2021, 1-6. [CrossRef]

20. Goldman, R.D.; Yan, T.D.; Seiler, M.; Cotanda, C.P.; Brown, J.C.; Klein, E.J.; Hoeffe, J.; Gelernter, R.; Hall, J.E.; Davis, A.L.; et al. Caregiver willingness to vaccinate their children against COVID-19: Cross sectional survey. Vaccine 2020, 38, 7668-7673. [CrossRef]

21. Bagateli, L.E.; Saeki, E.Y.; Fadda, M.; Agostoni, C.; Marchisio, P.; Milani, G.P. COVID-19 Vaccine Hesitancy among Parents of Children and Adolescents Living in Brazil. Vaccines 2021, 9, 1115. [CrossRef] 
22. Polack, F.P.; Thomas, S.J.; Kitchin, N.; Absalon, J.; Gurtman, A.; Lockhart, S.; Perez, J.L.; Marc, G.P.; Moreira, E.D.; Zerbini, C.; et al. Safety and efficacy of the BNT162b2 mRNA COVID-19 vaccine. N. Engl. J. Med. 2020, 383, 2603-2615. [CrossRef]

23. World Health Organization. Vaccine Efficacy, Effectiveness and Protection. 14 July 2021. Available online: https://www.who.int/ news-room/feature-stories/detail/vaccine-efficacy-effectiveness-and-protection (accessed on 4 November 2021).

24. Centers for Disease Control and Prevention (CDC). How Flu Vaccine Effectiveness and Efficacy are Measured. Available online: https:/ / www.cdc.gov/flu/vaccines-work/effectivenessqa.htm (accessed on 17 September 2021).

25. U.S. Food \& Drug Administration Development and Licensure of Vaccines to Prevent COVID-19. Available online: https://www. fda.gov/regulatory-information/search-fda-guidance-documents/development-and-licensure-vaccines-prevent-covid-19 (accessed on 17 September 2021).

26. AlShurman, B.A.; Khan, A.F.; Mac, C.; Majeed, M.; Butt, Z.A. What Demographic, Social, and Contextual Factors Influence the Intention to Use COVID-19 Vaccines: A Scoping Review. Int. J. Environ. Res. Public Health 2021, 18, 9342. [CrossRef]

27. Biswas, R.; Alzubaidi, M.S.; Shah, U.; Abd-Alrazaq, A.A.; Shah, Z. A Scoping Review to Find Out Worldwide COVID-19 Vaccine Hesitancy and Its Underlying Determinants. Vaccines 2021, 9, 1243. [CrossRef]

28. Bono, S.A.; Faria de Moura Villela, E.; Siau, C.S.; Chen, W.S.; Pengpid, S.; Hasan, M.T.; Sessou, P.; Ditekemena, J.D.; Amodan, B.O.; Hosseinipour, M.C.; et al. Factors Affecting COVID-19 Vaccine Acceptance: An International Survey among Low- and Middle-Income Countries. Vaccines 2021, 9, 515. [CrossRef]

29. IBM. IBM SPSS Statistics for Windows. Available online: https://www.ibm.com/products/spss-statistics (accessed on 4 November 2021).

30. Faul, F.; Erdfelder, E.; Buchner, A.; Lang, A.-G. Statistical power analyses using G*Power 3.1: Tests for correlation and regression analyses. Behav. Res. Methods 2009, 41, 1149-1160. [CrossRef]

31. Zhang, K.C.; Fang, Y.; Cao, H.; Chen, H.; Hu, T.; Chen, Y.Q.; Zhou, X.; Wang, Z. Parental Acceptability of COVID-19 Vaccination for Children Under the Age of 18 Years: Cross-Sectional Online Survey. JMIR Pediatr. Parent. 2020, 3, e24827. [CrossRef]

32. Bell, S.; Clarke, R.; Mounier-Jack, S.; Walker, J.L.; Paterson, P. Parents' and guardians' views on the acceptability of a future COVID-19 vaccine: A multi-methods study in England. Vaccine 2020, 38, 7789-7798. [CrossRef]

33. Yigit, M.; Ozkaya-Parlakay, A.; Senel, E. Evaluation of COVID-19 Vaccine Refusal in Parents. Pediatr. Infect. Dis. J. 2021, 40, e134-e136. [CrossRef]

34. Akıs, S.; Velıpasaoglu, S.; Camurdan, A.D.; Beyazova, U.; Sahın, F. Factors associated with parental acceptance and refusal of pandemic influenza A/H1N1 vaccine in Turkey. Eur. J. Pediatr. 2011, 170, 1165-1172. [CrossRef]

35. Karafillakis, E.; Larson, H.J. The benefit of the doubt or doubts over benefits? A systematic literature review of perceived risks of vaccines in European populations. Vaccine 2017, 35, 4840-4850. [CrossRef]

36. Karlsson, L.C.; Soveri, A.; Lewandowsky, S.; Karlsson, L.; Karlsson, H.; Nolvi, S.; Karukivi, M.; Lindfelt, M.; Antfolk, J. Fearing the disease or the vaccine: The case of COVID-19. Personal. Individ. Differ. 2021, 172, 110590. [CrossRef]

37. Sauer, M.; Vasudevan, P.; Meghani, A.; Luthra, K.; Garcia, C.; Knoll, M.D.; Privor-Dumm, L. Situational assessment of adult vaccine preventable disease and the potential for immunization advocacy and policy in low- and middle-income countries. Vaccine 2021, 39, 1556-1564. [CrossRef]

38. Szilagyi, P.G.; Shah, M.D.; Delgado, J.R.; Thomas, K.; Vizueta, N.; Cui, Y.; Vangala, S.; Shetgiri, R.; Kapteyn, A. Parents' Intentions and Perceptions About COVID-19 Vaccination for Their Children: Results from a National Survey. Pediatrics 2021, $148,148$. [CrossRef]

39. Ahinkorah, B.O.; Ameyaw, E.K.; Hagan, J.E.J.; Seidu, A.-A.; Schack, T. Rising Above Misinformation or Fake News in Africa: Another Strategy to Control COVID-19 Spread. Front. Commun. 2020, 5, 45. [CrossRef]

40. Mohd Hanafiah, K.; Wan, C.D. Public Knowledge, Perception and Communication Behavior Surrounding COVID-19 in Malaysia. 2020. Available online: https://www.researchgate.net/profile/Khayriyyah-Mohd-Hanafiah/publication/340568282_Public_ knowledge_perception_and_communication_behavior_surrounding_COVID-19_in_Malaysia/links/5eaf9cc4299bf18b95948 $\mathrm{fb} 0 /$ Public-knowledge-perception-and-communication-behavior-surrounding-COVID-19-in-Malaysia.pdf (accessed on 4 November 2021).

41. Torun, S.D.; Torun, F.; Catak, B. Healthcare workers as parents: Attitudes toward vaccinating their children against pandemic influenza A/H1N1. BMC Public Health 2010, 10, 596. [CrossRef]

42. Prado, B. COVID-19 in Brazil: "So what?". Lancet 2020, 395, 1461. [CrossRef]

43. Balakrishnan, V.; Ng, K.S.; Rahim, H.A. To share or not to share-The underlying motives of sharing fake news amidst the COVID-19 pandemic in Malaysia. Technol. Soc. 2021, 66, 101676. [CrossRef]

44. Bish, A.; Yardley, L.; Nicoll, A.; Michie, S. Factors associated with uptake of vaccination against pandemic influenza: A systematic review. Vaccine 2011, 29, 6472-6484. [CrossRef] [PubMed]

45. Kulu, H.; Dorey, P.S. Infection rates from COVID-19 in Great Britain by geographical units: A model-based estimation from mortality data. Health Place 2021, 67, 102460. [CrossRef] [PubMed]

46. Paul, R.; Arif, A.A.; Adeyemi, O.; Ghosh, S.; Han, D. Progression of COVID-19 From Urban to Rural Areas in the United States: A Spatiotemporal Analysis of Prevalence Rates. J. Rural. Health 2020, 36, 591-601. [CrossRef]

47. Bocquier, A.; Fressard, L.; Cortaredona, S.; Zaytseva, A.; Ward, J.; Gautier, A.; Peretti-Watel, P.; Verger, P. Social differentiation of vaccine hesitancy among French parents and the mediating role of trust and commitment to health: A nationwide cross-sectional study. Vaccine 2018, 36, 7666-7673. [CrossRef] 
48. Swaney, S.E.; Burns, S. Exploring reasons for vaccine-hesitancy among higher-SES parents in Perth, Western Australia. Health Promot. J. Aust. 2018, 30, 143-152. [CrossRef]

49. Wilson, S.L.; Wiysonge, C. Social media and vaccine hesitancy. BMJ Glob. Health 2020, 5, e004206. [CrossRef]

50. Rubin, G.J.; Potts, H.; Michie, S. Likely uptake of swine and seasonal flu vaccines among healthcare workers. A cross-sectional analysis of UK telephone survey data. Vaccine 2011, 29, 2421-2428. [CrossRef]

51. Setbon, M.; Raude, J. Factors in vaccination intention against the pandemic influenza A/H1N1. Eur. J. Public Health 2010, 20, 490-494. [CrossRef]

52. Glanz, J.M.; Newcomer, S.R.; Narwaney, K.J.; Hambidge, S.J.; Daley, M.F.; Wagner, N.M.; McClure, D.L.; Xu, S.; Rowhani-Rahbar, A.; Lee, G.M.; et al. A Population-Based Cohort Study of Undervaccination in 8 Managed Care Organizations across the United States. JAMA Pediatr. 2013, 167, 274-281. [CrossRef]

53. Gowda, C.; Dempsey, A.F. The rise (and fall?) of parental vaccine hesitancy. Hum. Vaccines Immunother. 2013, 9, 1755-1762. [CrossRef]

54. Daley, M.F.; Crane, L.A.; Chandramouli, V.; Beaty, B.L.; Barrow, J.; Allred, N.; Berman, S.; Kempe, A. Influenza Among Healthy Young Children: Changes in Parental Attitudes and Predictors of Immunization During the 2003 to 2004 Influenza Season. Pediatrics 2006, 117, e268-e277. [CrossRef]

55. Elliott, C.; Farmer, K. Immunization status of children under 7 years in the Vikas Nagar area, North India. Child Care Health Dev. 2006, 32, 415-421. [CrossRef]

56. Zou, X.; Cao, B. COVID-19 vaccines for children younger than 12 years: Are we ready? Lancet Infect. Dis. 2021, 21, 1614-1615. [CrossRef]

57. Malik, A.A.; McFadden, S.M.; Elharake, J.; Omer, S.B. Determinants of COVID-19 vaccine acceptance in the US. E Clin. Med. 2020, 26, 100495. [CrossRef]

58. Brooks, S.K.; Webster, R.K.; Smith, L.E.; Woodland, L.; Wessely, S.; Greenberg, N.; Rubin, G.J. The psychological impact of quarantine and how to reduce it: Rapid review of the evidence. Lancet 2020, 395, 912-920. [CrossRef]

59. Wong, L.P.; Alias, H.; Wong, P.-F.; Lee, H.Y.; Abubakar, S. The use of the health belief model to assess predictors of intent to receive the COVID-19 vaccine and willingness to pay. Hum. Vaccines Immunother. 2020, 16, 2204-2214. [CrossRef] [PubMed]

60. Faasse, K.; Newby, J. Public Perceptions of COVID-19 in Australia: Perceived Risk, Knowledge, Health-Protective Behaviors, and Vaccine Intentions. Front. Psychol. 2020, 11, 551004. [CrossRef] [PubMed]

61. Disparities in the risk and outcomes of COVID-19. Public Health England Press: London, UK. 2020. Available online: https://assets.publishing.service.gov.uk/government/uploads/system/uploads/attachment_data/file/908434/Disparities_ in_the_risk_and_outcomes_of_COVID_August_2020_update.pdf (accessed on 4 November 2021).

62. Gallè, F.; Sabella, E.; Roma, P.; De Giglio, O.; Caggiano, G.; Tafuri, S.; Da Molin, G.; Ferracuti, S.; Montagna, M.; Liguori, G.; et al. Knowledge and Acceptance of COVID-19 Vaccination among Undergraduate Students from Central and Southern Italy. Vaccines 2021, 9, 638. [CrossRef] [PubMed]

63. Walter, E.B.; Talaat, K.R.; Sabharwal, C.; Gurtman, A.; Lockhart, S.; Paulsen, G.C.; Barnett, E.D.; Muñoz, F.M.; Maldonado, Y.; Pahud, B.A.; et al. Evaluation of the BNT162b2 COVID-19 Vaccine in Children 5 to 11 Years of Age. N. Engl. J. Med. 2021. [CrossRef] [PubMed]

64. Gerber, J.S.; Offit, P.A. COVID-19 vaccines for children. Science 2021, 374, 913. [CrossRef]

65. Goldman, R.D.; Bone, J.N.; Gelernter, R.; Krupik, D.; Ali, S.; Mater, A.; Thompson, G.C.; Yen, K.; Griffiths, M.A.; Klein, A.; et al. National COVID-19 vaccine program progress and parents' willingness to vaccinate their children. Hum. Vaccines Immunother. 2021, 1-7. [CrossRef] 